Pacific Journal of Mathematics

THE TIME-DOMAIN ANALYSIS OF A CONTINUOUS
PARAMETER WEAKLY STATIONARY STOCHASTIC PROCESS 


\section{THE TIME-DOMAIN ANALYSIS OF A CONTINUOUS PARAMETER WEAKLY STATIONARY STOCHASTIC PROCESS}

\section{P. MASANI AND J. ROBERTSON}

1. Introduction. In this paper we shall give a new, spectral-free, method to obtain the differential innovations and the Wold decomposition of a univariate, continuous parameter, weakly stationary ${ }^{1}$, mean-continuous, non-deterministic stochastic process $\left(f_{t},-\infty<t<\infty\right)$. We shall affect a transition from the continuous to the discrete parameter case by systematic use of the infinitesimal generator $i H$ of the shift group $\left(U_{t},-\infty<t<\infty\right)$ of the process, and of the Cayley transform $V$ of the self-adjoint operator $H(\S 2)$. Our analysis will be purely in the time-domain.

With the $f_{t}$-process we shall associate the discrete parameter process $\left(f_{n}^{\prime}\right)_{n=-\infty}^{\infty}$, where $f_{n}^{\prime}=V^{n}\left(f_{0}\right)$. Since $V$ is unitary, the $f_{n}^{\prime}$-process is weakly stationary. Letting $\mathscr{M}_{t}, \mathscr{C}_{n}^{\prime}$ be the past and present subspaces of the $f_{t^{-}}$and $f_{n}^{\prime}$-processes, respectively, and $\mathscr{C}_{-\infty}, \mathscr{C}_{-\infty}^{\prime}$ be their remote pasts, we shall show that $\mathscr{M}_{0}=\mathscr{M}_{0}^{\prime}$ and $\mathscr{M}_{-\infty}=\mathscr{M}_{-\infty}^{\prime}(\S 4)$. In the nondeterministic case we shall show that the subspace $\mathscr{N}_{t}=\mathscr{M}_{-\infty}^{\perp} \cap \mathscr{C}_{t}$ is the past and present of the process $\left(h_{t},-\infty<t<\infty\right)$, where $h_{t}=U_{t}\left(h_{0}^{\prime}\right), h_{0}^{\prime}$ being the 0 th normalized innovation of the discrete $f_{n}^{\prime}$-process $(\S 5)$. We shall then show $(\S 6)$ that the $h_{t}$-process is weakly Markovian ${ }^{1}$ with covariance $e^{-|t|}$ for lag $t$, and that if

$$
\xi_{t}=T_{t}\left(h_{0}^{\prime}\right), \quad \text { where } \quad T_{t}=\frac{1}{\sqrt{2}}\left\{U_{t}-I+\int_{0}^{t} U_{s} d s\right\},
$$

the process $\left(\xi_{t},-\infty<t<\infty\right)$ has stationary, orthogonal increments such that $\left|\xi_{b}-\xi_{a}\right|^{2}=|b-a|$. These increments are the "differential innovations" of our $f_{t}$-process; for we shall show (6.6) that the set of all convergent stochastic integrals $\int_{-\infty}^{t} c(s) d \xi_{s}, c \in L_{2}(-\infty, t)$, is identical with the subspace $\mathscr{N}_{t}$ mentioned above. Since

$$
\mathscr{M}_{t}=\mathscr{N}_{t}+\mathscr{M}_{-\infty}, \mathscr{N}_{t} \perp \mathscr{M}_{-\infty},
$$

it follows at once that $f_{t}=u_{t}+v_{t}$, where the $u_{t}$ form a one-sided moving

Received March, 21, 1962. Preliminary attempts in the direction of the research here presented were made by the first-named author some years ago in collaboration with Mr. P. D. Kapadia of Bombay, India, whose help we gratefully acknowledge. Much of the work was done recently at Indiana University, that of the first named author being supported in part by the Office of Naval Research.

1 In this paper the term "weakly" has the same meaning as Doob's expression "in the wide sense" [5, p. 95]. 
average process, and the $v_{t}$ a deterministic one:

$$
u_{t}=\int_{0}^{\infty} c(s) d_{s} \xi_{t-s}, \quad v_{t}=\text { projection of } f_{t} \text { on } \mathscr{M}_{-\infty} \text {. }
$$

We thus get the Wold decomposition ef. 6.7 below.

In justification of this new approach we may mention its simplicity and coherence. With the time-domain analysis so completed, one can develop the spectral theory in an equally coherent way. One can also deal conveniently with the extension to vector-valued processes. In comparison, an approach in which spectral considerations are brought to bear on time-domain questions or vice versa seems cumbersome and roundabout. But quite apart from this, our approach is essentially more general than one based on the spectral resolution of the group $\left(U_{t},-\infty<t<\infty\right)$ and is more suggestive of further research, although it does not yield any really new results on univariate stationary processes. As prediction theory has advanced, its connection with the theory of shift-invariant subspaces of the Hardy class $\mathrm{H}_{2}$ initiated by Beurling [2] has been noticed; see especially Helson and Lowdenslager [10] and Lax [14]. Recently Halmos [8] has brought to light a result, which shows that underlying both theories is a semi-group of isometries on a Hilbert space (cf. also [15]). (In the case under discussion, this semigroup comprises the (isometric) restrictions of the unitary operators $U_{t}^{*}$ to the subspace $M_{0}$.) One of us [16] has found that our approach based on use of the infinitesimal generator $i H$ and of the operator $T_{t}$ defined in (1.1) extends to general continuous parameter semi-groups of isometries to yield valuable results concerning their structure. But since in general these isometries will be non-normal, the generator $H$ will not be self-adjoint and the usual spectral considerations will fail; cf. Cooper [3]. Thus it seems worthwhile to try to dispense with spectral tools in the analysis of time-domain problems.

Hanner [9] was the first to make a purely time-domain analysis in the continuous parameter case. By an ingenious construction he proved the existence of differential innovations and derived the Wold decomposition. His approach, somewhat $a d$ hoc in nature, has not been pursued in the literature, and its points of contact with the earlier work of Cooper [3] have gone unnoticed. Our approach differs from that of Hanner and Cooper in the transition we make to the discrete parameter case by means of the infinitesimal generator and the Cayley transform.

It is reasonably clear that our approach will work in the case of processes for which the differential innovations can be had by Hanner's method. As an instance we cite the study of continuous parameter random distributions due to $\mathrm{K}$. Ito, Gelfand, and Balagangadharan [12, $7,1]$. It is also possible that our ideas may apply to some of the nonstationary processes studied recently by Cramer $\left[4,4^{\prime}, 4^{\prime \prime}\right]$. 
2. The infinitesimal generator and Cayley transform. Let $\left(U_{t},-\infty<t<\infty\right)$ be a strongly continuous group of unitary operators acting on a complex Hilbert space $\mathfrak{x}$; i.e. let

$$
\left\{\begin{array}{l}
\text { (a) } U_{t} \text { be a unitary operator on } \mathfrak{X} \text { onto } \mathfrak{X},-\infty<t<\infty . \\
\text { (b) } U_{s} U_{t}=U_{s+t}=U_{t} U_{s},-\infty<s, t<\infty . \\
\text { (c) } \left.U_{t+h} \rightarrow U_{t} \text { (strongly) }\right)^{2} \text { on } \mathfrak{X} \text { as } h \rightarrow 0,-\infty<t<\infty .
\end{array}\right.
$$

It is known [17, p. 385] that the group has an infinitesimal generator

$$
i H=\lim _{h \rightarrow 0} \frac{1}{h}\left\{U_{t}-I\right\} \quad \text { on } \mathscr{D},
$$

where $H$ is a self-adjoint operator with domain $\mathscr{D}$, and $\mathscr{D}$ is a linear manifold everywhere dense in $\mathfrak{X}$. Also, cf. [19, p. 142 and 6, p. 622]

$$
\left\{\begin{array}{l}
\text { (a) } H+i I \text { is one-to-one on } \mathscr{D} \text { onto } \mathfrak{X}, \\
\text { (b) }(H+i I)^{-1}=\frac{1}{i} \int_{0}^{\infty} e^{-t} U_{t} d t \text { is bounded and one-to-one } \\
\text { on } \mathfrak{X} \text { onto } \mathscr{D} \text {, and }\left|(H+i I)^{-1}\right|_{B} \leqq 1^{(3)} .
\end{array}\right.
$$

Now let $V$ be the Cayley transform of $H$ :

$$
V=c(H)=(H-i I)(H+i I)^{-1} \text { on } \mathfrak{X} .
$$

Then [19, p. 304]

$$
\left\{\begin{array}{l}
\text { (a) } V \text { is unitary on } \mathfrak{X} \text { onto } \mathfrak{X}, \\
\text { (b) } I-V=2 i(H+i I)^{-1} \text { is one-to-one on } \mathfrak{X} \text { onto } \mathscr{D}, \\
\text { (c) } H=i(I+V)(I-V)^{-1} \text { on } \mathscr{D}, \\
\text { (d) } U_{t} V^{n}=V^{n} U_{t} \text { on } \mathfrak{X},-\infty<n, t<\infty, n=\text { integer. }
\end{array}\right.
$$

In this section we shall establish the relationship between $U_{t}$ and $V^{n}$ for arbitrary $t$ and $n$ on which will hinge the subsequent development.

The $U_{t}$ are expressible in terms of $H$ by the Hille-Yosida exponential formula, cf. [17, p. 403],

$$
\left\{\begin{array}{l}
\left.U_{t}=\lim _{n \rightarrow \infty} \exp \left(t i H J_{n}\right), \text { (strong) }\right)^{2}, \quad t \geqq 0 \\
J_{n}=\left(I-\frac{1}{h} i H\right)^{-1} .
\end{array}\right.
$$

One sees trivially that $J_{n}$ is a bounded operator and that so therefore is $i H J_{n}=n\left(J_{n}-I\right)$. Hence the term $\exp \left(t i H J_{n}\right)$ in (2.6) is definable

${ }^{2}$ It is to be understood in the sequel that all operator-limits are in the strong sense.

${ }^{3}|T|_{B}$ refers to the Banach norm of the operator $T$. 
by the usual power-series. We now assert two lemmas:

2.7 LeMma. (Expression of $U_{t}$ in terms of $V^{k}$ ).

$$
U_{ \pm t}=e^{-t} I+\lim _{n \rightarrow \infty} \sum_{k=1}^{\infty} \frac{1}{k !}\left(\frac{-n t}{n+1}\right)^{k}\left\{\left(I+A_{ \pm n}\right)^{k}-I\right\}, \quad t \geqq 0,
$$

where

$$
A_{ \pm n}=\frac{2 n}{n+1} \sum_{j=1}^{\infty}\left(\frac{n-1}{n+1}\right)^{\jmath-1} V^{ \pm j}, \quad n \geqq 0 .
$$

Proof. Let $t \geqq 0$. Then by (2.6)

$$
U_{t}=\lim _{n \rightarrow \infty} \sum_{k=0}^{\infty} \frac{t^{k}}{k !}\left(i H J_{n}\right)^{k} \text {. }
$$

Using (2.5)(c) we can express the R.H.S. of (1) in terms of $V$ :

$$
\begin{aligned}
i H J_{n} & =-(I+V)(I-V)^{-1}\left\{I+\frac{1}{n}(I+V)(I-V)^{-1}\right\}^{-1} \\
& =-\frac{n}{n+1}\left(I+A_{n}\right)
\end{aligned}
$$

after some simplification. Thus

$$
\left(i H J_{n}\right)^{k}=\left(-\frac{n}{n+1}\right)^{k} I+\left(-\frac{n}{n+1}\right)^{k}\left\{\left(I+A_{n}\right)^{k}-I\right\}, \quad k \geqq 0 .
$$

Hence from (1)

(2) $U_{t}=\lim _{n \rightarrow \infty} \exp \left(\frac{-n t}{n+1}\right) I+\lim _{n \rightarrow \infty} \sum_{k=1}^{\infty} \frac{1}{k !}\left(\frac{-n t}{n+1}\right)^{k}\left\{\left(I+A_{n}\right)^{-1}-I\right\}$.

Since the first term on the R.H.S. is $e^{-t} I$, we have the desired expression for $U_{t}, t \geqq 0$.

To obtain the expression for $U_{-t}, t \geqq 0$, we note that $U_{-t}=U_{t}^{*}$, $V^{*}=V^{-1}$ and so $A_{n}^{*}=A_{-n}, n \geqq 0$. Thus, taking adjoints on both sides of (2), we get the desired result.

2.8 Lemma. (Expression of $V^{n}$ in terms of $U^{t}$ ).

$$
V^{ \pm n}=I+2 \int_{0}^{\infty} L_{n}^{\prime}(2 t) e^{-t} U_{ \pm t} d t, \quad n \geqq 0
$$

where

$$
L_{n}(t)=\sum_{k=0}^{n} \frac{(-1)^{k}}{k !}\left(\begin{array}{l}
n \\
k
\end{array}\right) t^{k}, \quad n \geqq 0,(n \text {th Laguerre polynomial }) .
$$


Proof. The result obviously holds for $n=0$. We establish it for $n>0$ by induction. For $n=1$, the desired result reduces to the equality

$$
V=I-2 \int_{0}^{\infty} e^{-t} U_{t} d t
$$

the correctness of which is clear from (2.5)(b) 'and (2.5)(b). Next, assuming the result for $n$, we find using (1) that

$$
V^{n+1}=I+2 \int_{0}^{\infty}\left\{L_{n}^{\prime}(2 t)-1\right\} e^{-t} U_{t} d t-4 \int_{0}^{\infty} \int_{0}^{\infty} L_{n}^{\prime}(2 t) e^{-(s+t)} U_{s+t} d s d t .
$$

Putting $\sigma=s+t$, and using Dirichlet's formulae we find that

$$
4 \int_{0}^{\infty} \int_{0}^{\infty} L_{n}^{\prime}(2 t) e^{-(s+t)} U_{s+t} d s d t=2 \int_{0}^{\infty}\left\{L_{n}(2 \sigma)-1\right\} e^{-\sigma} U_{\sigma} d \sigma
$$

Hence

$$
V^{n+1}=I+2 \int_{0}^{\infty}\left\{L_{n}^{\prime}(2 t)-L_{n}(2 t)\right\} e^{-t} U_{t} d t .
$$

Since the Laguerre polynomials satisfy the recurrence relation $L_{n}=$ $L_{n}^{\prime}-L_{n+1}^{\prime}$, cf. [18, p. 299, (10)] we get

$$
V^{n+1}=I+2 \int_{0}^{\infty} L_{n+1}^{\prime}(2 t) e^{-t} U_{t} d t
$$

as desired. The result thus holds for all $n \geqq 1$. Its validity for $n \leqq$ -1 follows on taking adjoints and noting that $V^{-n}=\left(V^{n}\right)^{*}$ and $U_{-t}=$ $U_{t}^{*}$.

We shall denote by $\mathfrak{S}\left(X_{\lambda}\right)_{\lambda \in \Lambda}$ the (closed) subspace spanned by the subsets $X_{\lambda}$ of $\mathfrak{X}$, for $\lambda \in \Lambda$. We now assert the following lemma:

2.9 Lemma. For any $X \subseteq \mathfrak{X}$, we have

$$
\begin{aligned}
& \mathfrak{S}\left\{V^{-n}(X)\right\}_{n \geqq 0}=\mathscr{S}\left\{U_{-t}(X)\right\}_{t \geqq 0} \\
& \Im\left\{V^{n}(X)\right\}_{n \geqq 0}=\Im\left\{U_{t}(X)\right\}_{t \geqq 0} \text {. }
\end{aligned}
$$

Proof (a). Lemma 2.8 asserts that for $n \geqq 0 \quad V^{-n}$ is the strong limit of a linear combination of the $U_{-t}$ for $t \geqq 0$. It follows that for any $X \subseteq \mathfrak{X}$,

$$
V^{-n}(X) \subseteq \subseteq\left\{U_{-t}(X)\right\}_{t \geqq 0},
$$

whence

$$
\Im\left\{V^{-n}(X)\right\}_{n \geqq 0} \subseteq \subseteq\left\{U_{-t}(X)\right\}_{t \geqq 0} .
$$

On the other hand, Lemma 2.7 asserts that for $t \geqq 0, U_{-t}$ is the strong 
limit of a linear combination of the $V^{-n}$, for $n \geqq 0$, so that

$$
U_{-t}(X) \subseteq \subseteq\left\{V^{-n}(X)\right\}_{n \geqq 0} .
$$

From this follows the inclusion reverse to (1), thereby yielding (a).

(b) can be derived similarly from Lemmas 2.7, 2.8 taking $V^{n}, U_{t}$, instead of $V^{-n}, U_{-t}$, with $n, t \geqq 0$.

3. Weakly stationary stochastic processes. In this section we shall recall the basic notions and results on weakly stationary stochastic processes.

By a weakly stationary stochastic process (S.P.) is meant a function $f$ on $(-\infty, \infty)$ to a complex Hilbert space $X$ such that the inner product

$$
\left(f_{s}, f_{t}\right)=\gamma_{s-t}
$$

depends only on the difference $s-t$ and not on $s$ and $t$ separately. The complex-valued function $\gamma$ on $(-\infty, \infty)$ is called the covariance function of the S.P. It is convenient to denote the values of $f$ and $\gamma$ at $t$ by $f_{t}$ and $\gamma_{t}$ rather than by $f(t)$ and $\gamma(t)$, and to denote the S.P. itself by $\left(f_{t},-\infty<t<\infty\right)$ rather than by $f$.

We shall be especially interested in the subspaces

$$
\left\{\begin{array}{l}
\mathscr{M}_{t}=\mathfrak{S}\left(f_{s}\right)_{s \leqq t}, \quad-\infty<t<\infty \\
\mathscr{M}_{-\infty}=\bigcap_{-\infty<t<\infty} \mathscr{M}_{t}, \quad \mathscr{M}_{\infty}=\subseteq\left(f_{s}\right)_{s<\infty} .
\end{array}\right.
$$

We shall call $\mathscr{M}_{t}$ the past and present of $f_{t}, \mathscr{M}_{-\infty}$ the remote past of the S.P., and $\mathscr{C}_{\infty}$ the space spanned by the S.P. Obviously

$$
\left\{\begin{array}{l}
\mathscr{M}_{-\infty} \subseteq \mathscr{M}_{s} \subseteq \mathscr{M}_{t} \subseteq \mathscr{M}_{\infty}, \quad-\infty<s<t<\infty \\
\mathscr{M}_{-\infty}=\bigcap_{t \leqq 0} \mathscr{M}_{t} .
\end{array}\right.
$$

It is known, cf. Karhunen [13, p. 55], that if $\left(f_{t},-\infty<t<\infty\right)$ is a weakly stationary S.P., then there exists a group of unitary operators $U_{t}$ on $\mathfrak{X},-\infty<t<\infty$, such that

$$
f_{s+t}=U_{t}\left(f_{s}\right), \quad-\infty<s, t<\infty .
$$

The operators $U_{t}$ are uniquely determined on the subspace $\mathscr{M}_{\infty}$ but not on $X$. We shall call $\left(U_{t},-\infty<t<\infty\right)$ the shift group of the S.P. $\left(f_{t},-\infty<t<\infty\right)$. It follows easily, cf. Hanner [9, p.162], that

$$
U_{t}\left(\mathscr{M}_{s}\right)=\mathscr{M}_{s+t}, U_{t}\left(\mathscr{M}_{-\infty}\right)=\mathscr{C}_{-\infty}, U_{t}\left(\mathscr{M}_{\infty}\right)=\mathscr{C}_{\infty},-\infty<s, t<\infty .
$$

We call a S.P. $\left(f_{t},-\infty<t<\infty\right)$ mean-continuous, if the function $f$ is continuous on $(-\infty, \infty)$ with respect to the metric induced by the norm of the Hilbert space $\mathfrak{X}$. From the stationarity condition (3.1) we 
readily infer the following:

3.6 Lemma. For a weakly stationary S.P. $\left(f_{t},-\infty<t<\infty\right)$ with covariance function $\gamma$ mean-continuity is equivalent to each of the conditions:

(i) $f$ is continuous at 0 ,

(ii) $\gamma$ is continuous at 0 ,

(iii) $\gamma$ is continuous on $(-\infty, \infty)$,

(iv) the shift group $\left(U_{t},-\infty<t<\infty\right)$ is strongly continuous on $\mathscr{C l}_{\infty}$.

The following result is known:

3.7 Lemma. If the S.P. is mean-continuous, then

(a) $\mathscr{C}_{\infty}$ is a separable subspace of $\mathscr{X}$,

(b) $\mathscr{C l}_{t-}=\mathscr{H}_{t}=\mathscr{H}_{t+},-\infty<t<\infty$, where $\mathscr{M}_{t^{-}}=$clos. $\mathrm{U}_{s<t} \mathscr{H}_{s}$, $\mathscr{C l}_{t+}=\bigcap_{s>t} \mathscr{K}_{s}$.

4. The associated discrete parameter process. Let $\left(f_{t},-\infty<t<\infty\right)$ be a weakly stationary, mean-continuous S.P. with shift group $\left(U_{t}\right.$, $-\infty<t<\infty)$. Let $V$ be the Cayley transform of $H$, where $i H$ is the infinitesimal generator of the shift group, cf. (2.2), (2.4). Let

$$
f_{n}^{\prime}=V^{n}\left(f_{0}\right) \text {. }
$$

Then the bisequence $\left(f_{n}^{\prime}\right)_{-\infty}^{\infty}$ is a discrete-parameter, weakly stationary S.P. with shift operator $V$. We shall call it the discrete S.P. associated with $\left(f_{t},-\infty<t<\infty\right)$.

We shall denote the past and present of $f_{n}^{\prime}$, the remote past, and the subspace spanned by the S.P. $\left(f_{n}^{\prime}\right)_{-\infty}^{\infty}$ by $\mathscr{C}_{n}^{\prime}, \mathscr{C}_{-\infty}^{\prime}$, and $\mathscr{M}_{\infty}^{\prime}$, respectively; thus

$$
\mathscr{M}_{n}^{\prime}=S\left(f_{i}^{\prime}\right)_{k=-\infty}^{n}, \quad \mathscr{C}_{-\infty}^{\prime}=\bigcap_{n=\infty} \mathscr{M}_{n}^{\prime}, \quad \mathscr{C}_{\infty}^{\prime}=\mathscr{S}\left(f_{k}^{\prime}\right)_{k=-\infty}^{\infty} .
$$

It follows that

$$
\left\{\begin{array}{l}
\mathscr{H}_{-\infty}^{\prime} \subseteq \mathscr{K}_{m}^{\prime} \subseteq \mathscr{C}_{n}^{\prime} \leqq \mathscr{K}_{\infty}^{\prime},-\infty<m<n<\infty \\
\mathscr{H}_{-\infty}^{\prime}=\bigcap_{n=-\infty} \mathscr{H}_{n}^{\prime} .
\end{array}\right.
$$

As far as we know the associated discrete parameter S.P. $\left(f_{n}^{\prime}\right)_{-\infty}^{\infty}$ has been defined in the literature, not by (4.1), but as the process whose spectral distribution is the Cayley transform (in the complex plane) of the spectral distribution of the given continuous parameter process, cf. e.g. Doob [5, p. 583]. It can be shown that the two definitions are equivalent. But as indicated in $\S 1$ there are advantages in adopting 
a purely time-domain and spectral-free definition. For instance, in the light of Lemma 2.9 we can assert the following theorem, which reveals the close relationship between the two processes. Variants of parts (a), (b) of this theorem are know, cf. e.g. Doob [5, p. 583-84]; part (c) is new as for as we know.

4.4 TheoRem. (a) $\mathscr{M}_{0}=\mathscr{C}_{0}^{\prime}$, (b) $\mathscr{C l}_{\infty}=\mathscr{C}_{\infty}^{\prime}$, (c) $\mathscr{C l}_{-\infty}=\mathscr{M}_{-\infty}^{\prime}$.

Proof. (a) Take $X=\left\{f_{0}\right\}$ in 2.9(a). We then get

$$
\mathscr{H}_{0}^{\prime}=\mathfrak{S}\left(V^{-n}\left(f_{0}\right)\right)_{n \geqq 0}=\mathfrak{S}\left(U_{-t}\left(f_{0}\right)\right)_{t \geqq 0}=\mathscr{C}_{0} .
$$

(b) Now take $X=\left\{f_{0}\right\}$ in $2.9(\mathrm{~b})$. We then get $\mathfrak{S}\left(V^{n}\left(f_{0}\right)\right)_{n \geqq 0}=$ $\subseteq\left(U_{t}\left(f_{0}\right)\right)_{t \geqq 0}$. Hence,

$$
\begin{aligned}
\mathscr{C}_{\infty}^{\prime} & =\operatorname{clos} .\left\{\mathscr{C}_{0}^{\prime}+\mathfrak{S}\left(V^{n}\left(f_{0}\right)\right)_{n \geqq 0}\right\} \\
& =\operatorname{clos} .\left\{\mathscr{C l}_{0}+\mathfrak{S}\left(U_{t}\left(f_{0}\right)\right)_{t \geq 0}\right\} \\
& =\mathscr{C}_{\infty} .
\end{aligned}
$$

(c) Take $X=\mathscr{H}_{-\infty}$ in 2.9(b). Then using (3.5) we get

$$
V^{k}\left(\mathscr{C}_{-\infty}\right) \subseteq \subseteq\left(V^{n}\left(\mathscr{M}_{-\infty}\right)\right)_{n \geqq 0}=\subseteq\left(U_{t}\left(\mathscr{C}_{-\infty}\right)\right)_{t \geqq 0}=\mathscr{M}_{-\infty}, \quad k \geqq 0 .
$$

Applying $V^{-k}$ to both sides, and using (a),

$$
\mathscr{C l}_{-\infty} \subseteq V^{-k}\left(\mathscr{C l}_{-\infty}\right) \subseteq V^{-k}\left(\mathscr{L C}_{0}\right)=V^{-k}\left(\mathscr{M}_{0}^{\prime}\right)=\mathscr{C l}_{-k}^{\prime}, \quad k \geqq 0 .
$$

Hence, cf. (4.3),

$$
\mathscr{L}_{-\infty} \subseteq \bigcap_{k=0}^{\infty} \mathscr{C}_{-k}^{\prime}=\mathscr{C}_{-\infty}^{\prime}
$$

Next taking $X=\mathscr{M}_{-\infty}^{\prime}$ in $2.9(\mathrm{~b})$, we get

$$
U_{s}\left(\mathscr{C}_{-\infty}^{\prime}\right) \subseteq \subseteq\left(U_{t}\left(\mathscr{M}_{-\infty}^{\prime}\right)\right)_{t \geqq 0}=S\left(V^{n}\left(\mathscr{M l}_{-\infty}^{\prime}\right)\right)_{n \geqq 0}=\mathscr{C}_{-\infty}^{\prime}, \quad s \geqq 0 .
$$

Proceeding as before, we derive the inclusion relation reverse to that in (1). Thus (c).

5. Non-deterministic S.P. Pre-Wold decomposition. We shall say that a S.P. $\left(f_{t},-\infty<t<\infty\right)$ is deterministic, if and only if $\mathscr{C}_{-\infty}=\mathscr{M}_{\infty}$; otherwise non-deterministic. From the stationarity condition (3.1) we infer the following lemma, cf. Hanner [9, p. 163]:

5.1 Lemma. For a weakly stationary S.P. the following conditions are equivalent:

(i) the S.P. is deterministic

(ii) $\mathscr{K}_{s}=\mathscr{C l}_{t}$ for all $s, t,-\infty<s, t<\infty$ 
(iii) $\mathscr{M}_{s}=\mathscr{M}_{t}$ for some $s, t-\infty<s<t<\infty$

(iv) $f_{t} \in \mathscr{M}_{s}$ for some $s, t,-\infty<s<t<\infty$.

Let the S.P. be non-deterministic. Then by 5.1 (iii) for any $t$ and any $s<t, \mathscr{M}_{-\infty} \subseteq \mathscr{M}_{s} \subset \mathscr{M}_{t}$. Hence

$$
\mathscr{N}_{t}^{2}=\mathscr{C}_{-\infty}^{\perp} \cap \mathscr{H}_{t} \neq\{0\}, \quad-\infty<t<\infty,
$$

and we get the decomposition

$$
\mathscr{K}_{t}=\mathscr{C l}_{-\infty}+\mathscr{N}_{t}, \quad \mathscr{C l}_{-\infty} \perp \mathscr{N}_{t} \neq\{0\}, \quad-\infty<t<\infty .
$$

Moreover from (3.5)

$$
U_{t}\left(\mathscr{N}_{s}\right)=\mathscr{N}_{s+t},-\infty<s, t<\infty .
$$

If in the preceding paragraphs of this section we interpret $s, t$ as integers rather than as real numbers, we get the definition and properties of non-deterministic processes in the discrete parameter case. But in the discrete case, additional results are readily available. We recall some of these in the next paragraph.

Let $\left(f_{n}^{\prime}\right)_{-\infty}^{\infty}$ be any weakly stationary, non-deterministic S.P. with shift operator $V$. Denote by $\left(f_{n}^{\prime} \mid \mathscr{M}_{n-1}^{\prime}\right)$ the orthogonal projection of $f_{n}^{\prime}$ on the subspace $\mathscr{C}_{n-1}^{\prime}$, cf. (4.2). Then

$$
g_{n}^{\prime}=f_{n}^{\prime}-\left(f_{n}^{\prime} \mid \mathscr{M}_{n-1}^{\prime}\right) \neq 0, \quad-\infty<n<\infty .
$$

The vectors $g_{n}^{\prime}$ and $h_{n}^{\prime}=g_{n}^{\prime}|| g_{n}^{\prime} \mid$ are called the $n$th innovation and normalized innovation vectors, respectively, of the process $\left(f_{n}^{\prime}\right)_{-\infty}^{\infty}$. It is easily seen that

$$
\left(h_{m}^{\prime}, h_{n}^{\prime}\right)=\delta_{m n}, \quad h_{m+n}^{\prime}=V^{m}\left(h_{n}^{\prime}\right), \quad-\infty<m, n<\infty,
$$

so that $\left(h_{n}^{\prime}\right)_{-\infty}^{\infty}$ is an orthonormal S.P. with the same shift operator $V$ as $\left(f_{n}^{\prime}\right)_{-\infty}^{\infty}$. It is an important fact that in the discrete analogue of (5.2), viz.

$$
\mathscr{C}_{n}^{\prime}=\mathscr{C}_{-\infty}^{\prime}+\mathscr{N}_{n}^{\prime}, \quad \mathscr{K}_{-\infty}^{\prime} \perp \mathscr{N}_{n}^{\prime} \neq\{0\},
$$

the subspace $N_{n}^{\prime}$ is the past and present of $h_{n}^{\prime}$ :

$$
\mathscr{N}_{n}^{\prime}=\mathfrak{S}\left(h_{k}^{\prime}\right)_{k=-\infty}^{n}=\mathfrak{S}\left(V^{k}\left(h_{0}^{\prime}\right)\right)_{k=-\infty}^{n} .
$$

The relations (5.6), (5.7) constitute the Wold decomposition of $M_{n}^{\prime}$. From this decomposition follows at once the canonical decomposition of $f_{n}^{\prime}$ into a one-sided moving-average part and a deterministic part:

$$
\left\{\begin{array}{l}
f_{n}^{\prime}=u_{n}^{\prime}+v_{n}^{\prime}, \quad-\infty<n<\infty \\
u_{n}^{\prime}=\left(f_{n}^{\prime} \mid \mathscr{N}_{-\infty}^{\prime}\right)=\sum_{k=0}^{\infty} c_{k} h_{n-k}^{\prime}, \quad c_{0}=\left|g_{0}^{\prime}\right|, \quad \sum_{k=0}^{\infty}\left|c_{k}\right|^{2}<\infty \\
v_{n}^{\prime}=\left(f_{n}^{\prime} \mid \mathscr{C}_{n}^{\prime}\right) \\
\left(u_{n}^{\prime}\right)_{-\infty}^{\infty},\left(v_{n}^{\prime}\right)_{-\infty}^{\infty} \text { have the same shift operator } V \text { as }\left(f_{n}^{\prime}\right)_{-\infty}^{\infty} .
\end{array}\right.
$$


To revert to the continuous parameter case, let $\left(f_{t},-\infty<t<\infty\right)$ be a weakly stationary, mean-continuous, non-deterministic S.P. with shift group $\left(U_{t},-\infty<t<\infty\right)$. It is clear from the equalities in 3.7(b) that attempts to define "innovation vectors" $g_{t}$ for this process by an equation analogous to (5.4) will fail. Indeed, since there is no atomic time unit in the continuous parameter case, all that we may expect our $f_{t}$-process to possess are "differential innovations."

Now let $\left(f_{n}^{\prime}\right)_{-\infty}^{\infty}$ be the discrete S.P. associated with $\left(f_{t},-\infty<t<\infty\right)$. Since the latter process is non-deterministic, it follows from Theorem 4.4 that so is the former. Let $h_{0}^{\prime}$ be its 0 th normalized innovation vector, and let

$$
h_{t}=U_{t}\left(h_{0}^{\prime}\right), \quad-\infty<t<\infty .
$$

The resulting process $\left(h_{t},-\infty<t<\infty\right)$ plays an important role in the theory. In $\S 6$ we shall show that it is weakly Markovian, and explain how the differential innovations of the $f_{t}$-process can be had from it. ${ }^{4}$ Here we shall show that the subspaces $\mathscr{N}_{t}$ of (5.2) are its past and present subspaces:

5.10 Theorem. (Pre-Wold Decomposition) Let $\left(f_{t},-\infty<t<\infty\right)$ be a weakly stationary, mean-continuous, non-deterministic S.P. with shift group $\left(U_{t},-\infty<t<\infty\right)$, so that $c f$. (5.2)

$$
\mathscr{M}_{t}=\mathscr{M}_{-\infty}+\mathscr{N}_{t}, \mathscr{M}_{-\infty} \perp \mathscr{N}_{t},
$$

Then $\mathscr{N}_{t}=\mathfrak{S}\left(h_{s}\right)_{s \leqq t}$ is the past and present of $h_{t},-\infty<t<\infty$.

Proof. By Theorem 4.4, $\mathscr{C}_{0}^{\prime}=\mathscr{C}_{0}, \mathscr{C}_{-\infty}^{\prime}=\mathscr{C}_{-\infty}$. Hence, taking $t=0=n$ in (5.2), (5.6) we see that $\mathscr{N}_{0}^{\prime}=\mathscr{N}_{0}$. But taking $X=\left\{h_{0}^{\prime}\right\}$ in 2.9(a), where $h_{0}^{\prime}$ is the 0th normalized innovation of the associated discrete process, we find on using (5.7) that

$$
\mathscr{N}_{0}=\mathscr{N}_{0}^{\prime}=\subseteq\left(V^{k}\left(h_{0}^{\prime}\right)\right)_{k=-\infty}^{0}=\mathfrak{S}\left(U_{s}\left(h_{0}^{\prime}\right)\right)_{s \leqq 0} \text {. }
$$

Hence by (5.3) and (5.9)

$$
\mathscr{N}_{t}^{\prime}=U_{t}\left(\mathscr{N}_{0}\right)=\mathfrak{S}\left(U_{s}\left(h_{0}^{\prime}\right)\right)_{s \leqq t}=\mathfrak{S}\left(h_{s}\right)_{s \leqq t} .
$$

\section{Differential innovations and the Wold decomposition. Let}

4 The physical significance of the $h_{\iota}$-process has been indicated by Wiener and Wintner [20]. When $¥$ is the class of $L_{2}$-functions on a probability space $(\Omega, \mathscr{S}, P)$, and $t$ is the time, $h_{t}$ provides the weak (or wide sense) version of "random time", i.e. time as measured by a perfect clook which is subjected to Brownian fluctuations. More precisely, if

$$
y_{t}(\omega)=\exp \left[i \lambda\left\{t+a x_{t}(\omega)\right\}\right], \quad \omega \in \Omega
$$

where $\left(x_{t},-\infty<t<\infty\right)$ is the Brownian movement S.P., and $\lambda, a$ are constants such that $a \lambda=\sqrt{ } 2$, then the $y_{t^{-}}$and $h_{t}$-processes have the same wide sense properties. 
$\left(f_{t},-\infty<t \in \infty\right)$ be a weakly stationary, mean-continuous, non-deterministic S.P. with shift group $\left(U_{t},-\infty<t<\infty\right)$, and let $h_{0}^{\prime}$ be the 0th normalized innovation vector of the associated discrete process $\left(f_{n}^{\prime}\right)_{-\infty}^{\infty}$. In the next lemma we study the S.P. $\left(h_{t},-\infty<t<\infty\right)$ defined by (5.9), the present and past subspaces $\mathscr{N}_{t}$ of which have been mentioned in the Pre-Wold decomposition 5.10.

6.1 Lemma. (a) The $h_{t}$-process is weakly (or wide sense) Markovian; more fully,

$$
\left(h_{t} \mid \mathscr{N}_{s}\right)=e^{s-t} h_{s}, \quad-\infty<s<t<\infty
$$

depends only on the terminal vector $h_{s}$ of $\mathscr{N}_{s}=\Im\left(h_{\sigma}\right)_{\sigma \leqq s}$.

(b) Its convariance function $\gamma$ is given by $\gamma_{t}=e^{-|t|},-\infty<t<\infty$.

Proof. (a) Let $t \geqq 0$. Then by 2.7

$$
h_{t}=e^{-t} h_{0}^{\prime}+\lim _{n \rightarrow \infty} \sum_{k=1}^{n} \frac{1}{k !}\left(\frac{-n t}{n+1}\right)^{k}\left\{\left(I+A_{n}\right)^{k}-I\right\}\left(h_{0}^{\prime}\right)
$$

where

$$
A_{n}\left(h_{0}^{\prime}\right)=\frac{2 n}{n+1} \sum_{j=1}^{\infty}\left(\frac{n-1}{n+1}\right)^{\jmath-1} V^{j}\left(h_{0}^{\prime}\right)
$$

Since by (5.5) the $h_{j}^{\prime}=V^{s}\left(h_{0}^{\prime}\right)$ constitute an orthonormal process, we see that

$$
h_{t}=e^{-t} h_{0}^{\prime}+\eta_{t}, \quad \text { where } \eta_{t} \perp h_{0}^{\prime}, h_{-1}^{\prime}, \cdots, t \geqq 0 .
$$

It follows from (5.10) that $\eta_{t} \perp \mathscr{N}_{0}=\Im\left(h_{s}\right)_{s \leqq 0}$. Hence

$$
e^{-t} h_{0}^{\prime}=\left(h_{t} \mid \mathscr{N}_{0}^{-}\right), t \geqq 0 \text {. }
$$

On applying $U_{s}$ to both sides we get

$$
e^{-t} h_{s}=\left(h_{s+t} \mid \mathscr{N}_{s}^{r}\right), \quad-\infty<s<\infty, \quad t \geqq 0 .
$$

This reduces to the desired relation on changing the index.

(b) From (1) it follows at once that

$$
\gamma_{t}=\left(h_{s+t}, h_{s}\right)=e^{-t}, \quad t \geqq 0 .
$$

This in turn entails that $\gamma_{t}=e^{-|t|},-\infty<t<\infty$.

We shall now study the $\xi_{t}$-process mentioned in (1.1). By definition

$$
\xi_{t}=\frac{1}{\sqrt{2}}\left\{h_{t}-h_{0}+\int_{0}^{t} h_{s} d s\right\}, \quad-\infty<t<\infty .
$$


It follows at once that $\xi_{0}=0$ and

$$
\xi_{b}-\xi_{a}=\frac{1}{\sqrt{2}}\left\{h_{b}-h_{a}+\int_{a}^{b} h_{s} d s\right\},-\infty<a, b<\infty .
$$

6.4 THEOREM. (a) The $\xi_{t}$-process has increments which are stationary under the group $\left(U_{t},-\infty<t<\infty\right)$, i.e.

$$
U_{t}\left(\xi_{b}-\xi_{a}\right)=\xi_{b+t}-\xi_{a+t}, \quad-\infty<a, b, t<\infty .
$$

(b) The $\xi_{t}$-process has orthogonal increments, i.e.

$$
\xi_{b}-\xi_{a} \perp \xi_{a}-\xi_{c}, \text { if }-\infty<a<b \leqq c<d<\infty \text {. }
$$

(c) $\left|\xi_{b}-\xi_{a}\right|^{2}=|b-a|, \quad-\infty<a, b<\infty$.

(d) $\left(\xi_{b}-\xi_{a}, \xi_{d}-\xi_{c}\right)=\left|\xi_{b}-\xi_{c}\right|^{2}=b-c$, if $-\infty<a<c<b<d<\infty$.

Proof. (a) follows at once from (6.3) since $U_{t}\left(h_{s}\right)=h_{s+t}$.

(b) Let $a<b \leqq c<d$. Then from (6.3) and 6.1(b),

$$
\begin{aligned}
2\left(\xi_{b}-\xi_{a}, \xi_{a}-\xi_{c}\right)= & \left(h_{b}-h_{a}+\int_{a}^{b} h_{s} d s, \quad h_{a}-h_{c}+\int_{c}^{a} h_{t} d t\right) \\
= & \left\{e^{b-a}-e^{b-c}+\int_{c}^{a} e^{b-t} d t\right\}-\left\{e^{a-a}-e^{a-c}+\int_{c}^{a} e^{a-t} d t\right\} \\
& +\int_{a}^{b}\left\{e^{s-a}-e^{s-c}+\int_{c}^{a} e^{s-t} d t\right\} d s .
\end{aligned}
$$

Since the expression in each \{\} on the R.H.S. is zero, the result follows.

(c) First let $0=a<b$. Then from (6.3) and 6.1(b)

$$
\begin{aligned}
2\left|\xi_{b}-\xi_{0}\right|^{2}= & \left(h_{b}-h_{0}+\int_{0}^{b} h_{s} d s, \quad h_{b}-h_{0}+\int_{0}^{b} h_{t} d t\right) \\
= & \left\{1-e^{-b}+\int_{0}^{b} e^{t-b} d t\right\}-\left\{e^{-b}-1+\int_{0}^{b} e^{-t} d t\right\} \\
& +\int_{0}^{b}\left\{e^{s-b}-e^{-s}+\int_{0}^{b} e^{-|s-t|} d t\right\} d s \\
= & 2\left(1-e^{-b}\right)+0+\int_{0}^{b} \int_{0}^{b} e^{-|s-t|} d t d s .
\end{aligned}
$$

Since the last integral equals

$$
\int_{0}^{b}\left\{\int_{0}^{s} e^{t-s} d t+\int_{s}^{b} e^{s-t} d t\right\} d s=2 b+2\left(e^{-b}-1\right),
$$

it follows that $\left|\xi_{b}-\xi_{0}\right|^{2}=b$.

Next, let $-\infty<a<b<\infty$. Then by (a) $\xi_{b}-\xi_{a}=U_{a}\left(\xi_{b-a}-\xi_{0}\right)$, $b-a>0$, and so

$$
\left|\xi_{b}-\xi_{a}\right|^{2}=\left|\xi_{b-a}-\xi_{0}\right|^{2}=b-a .
$$


(c) is a simple consequence of (a), (b), the vertification of which we leave to the reader.

In view of the last theorem, the stochastic integral $\int_{-\infty}^{\infty} c(s) d \xi_{s}$ will exist for any complex-valued function $c \in L_{2}(-\infty, \infty)$, cf. Doob [5, Ch. IX, §2]. In the next lemma we shall show that the vector $h_{t}$ is expressible in terms of the $\xi_{s}$ by means of such an integral. In effect we shall invert the relation expressed in (6.2):

\subsection{LeMma. (Inversion formula)}

$$
h_{t}=\sqrt{2}\left\{\xi_{t}-\int_{-\infty}^{t} e^{s-t} \xi_{s} d s\right\}=\sqrt{2} \int_{-\infty}^{t} e^{s-t} d \xi_{s}, \quad-\infty<t<\infty .
$$

Proof. Since $h_{t}=U_{t}\left(h_{0}^{\prime}\right)$ and $U$ (as a function of $t$ ) is strongly continuous on $(-\infty, \infty)$, it follows that the vector-valued function $h$ is continuous for $t \in(-\infty, \infty)$, and therefore by (6.2) so is the function $\xi$. Hence the Riemann integral $\int_{a}^{b} e^{s-t} \xi_{s} d s$ exist for $-\infty<a<b \leqq t$. Moreover, since

$$
\left|\int_{a}^{b} e^{s-t} \xi_{s} d s\right| \leqq \int e^{s-t}\left|\xi_{s}\right| d s=\int_{a}^{b} e^{s-t} \sqrt{ } \cdot d s \text { or } \sqrt{s} \cdot d s
$$

the infinite integral $\int_{-\infty}^{t} e^{s-t} \xi_{s} d s$ converges.

Now consider the case $t=0$. We have from (6.2)

$$
\begin{aligned}
\sqrt{2} \int_{-\infty}^{0} e^{s}\left(\xi_{0}-\xi_{s}\right) d s & =-\int_{-\infty}^{0} e^{s}\left\{h_{s}-h_{0}+\int_{0}^{s} h_{\sigma} d \sigma\right\} d s \\
& =-\int_{-\infty}^{0} e^{s} h_{s} d s+h_{0}+\int_{-\infty}^{0} \int_{s}^{0} e^{s} h_{\sigma} d \sigma d s .
\end{aligned}
$$

Now by Dirichlet's formula the last integral equals

$$
\int_{-\infty}^{0} \int_{-\infty}^{0} e^{s} h_{\sigma} d s d \sigma=\int_{-\infty}^{0}\left\{\int_{-\infty}^{0} e^{s} d s\right\} h_{\sigma} d \sigma=\int_{-\infty}^{0} e^{\sigma} h_{\sigma} d \sigma
$$

Hence

$$
\sqrt{2} \int_{-\infty}^{0} e^{s}\left(\xi_{0}-\xi_{s}\right) d s=h_{0} .
$$

Since for any real $t, U_{t}\left(\xi_{0}-\xi_{s}\right)=\xi_{t}-\xi_{s+t}, U_{t}\left(h_{0}\right)=h_{t}$, we get the first equality in the lemma by applying $U_{t}$ to both sides of (1) and then changing variables.

The second equality follows on integrating by parts:

$$
\int_{-\infty}^{t} e^{s-t} d \xi_{s}=\left[e^{s-t} \xi_{s}\right]_{s \rightarrow-\infty}^{s=t}-\int_{-\infty}^{t} \xi_{s} d_{s}\left(e^{s-t}\right)=\xi_{t}-\int_{-\infty}^{t} e^{s-t} \xi_{s} d s .
$$


The use of integration by parts is justified as follows. In the first place, for $-\infty<a<t<\infty$ we have

$$
\int_{a}^{t} e^{s-t} d \xi_{s}=\left[e^{s-t} \xi_{s}\right]_{s=a}^{s=t}-\int_{a}^{t} \xi_{s} d_{s}\left(e^{s-t}\right) .
$$

This follows from the fact that for a continuous integrand the stochastic integral is a Riemann-Stieltjes integral (with vector-valued integrator $\xi_{s}$ ) and that for the latter, integration by parts is valid, cf. [5, p. $429(2.6)]$ and [11, p. 63 (3.31)]]. Next, the last integral in (2) is obviously equal to $\int_{a}^{t} \xi_{s} e^{s-t} d s$. Finally, since both $\int_{-\infty}^{t} e^{s-t} d \xi_{s}, \int_{-\infty}^{t} \xi_{s} e^{s-t} d s$ are known to exist, we can let $a \rightarrow-\infty$ in (2), cf. [5, p. $428(2.4)]$.

The formulae (6.2) and 6.5 together entail the following important result:

6.6 Lemma. For any real $t$, the past and present subspace $\mathscr{N}_{t}$ of $h_{t}$ is the set of all (convergent) stochastic integrals $\int_{-\infty}^{t} c(s) d \xi_{s}$, with complex-valued functions $c \in L_{2}(-\infty, t)$, i.e. $\mathscr{N}_{t}=\mathfrak{S}\left(\xi_{\sigma}-\xi_{\tau}\right)_{\sigma, \tau \leq t}$.

Proof. Denote by $\mathscr{N}_{t}^{(\xi)}$ the set of all such stochastic integrals. Let $-\infty<\tau \leqq t<\infty$. Then by 6.5

$$
h_{\tau}=\sqrt{2} \int_{-\infty}^{\tau} e^{s-\tau} d \xi_{s}=\int_{-\infty}^{\tau} c(s) d \xi_{s},
$$

where $c(s)=\sqrt{ } 2 e^{s-\tau}$ on $(-\infty, \tau]$ and $c(s)=0$ on $(\tau, t]$. Since $c \in L_{2}(-\infty, t]$, it follows that $h_{\tau} \in \mathscr{N}_{t}^{(\xi)}$. Hence $\mathscr{N}_{t}=\mathfrak{S}\left(h_{\tau}\right)_{\tau \leqq t} \subseteq \mathscr{N}_{t}^{(\xi)}$.

To prove the reverse inclusion, let

$$
g=\int_{-\infty}^{t} c(s) d \xi_{s}, \text { where } c \in L_{2}(-\infty, t] .
$$

Suppose first that $c$ is a step-function:

$$
c(s)=\sum_{k=1}^{n} c_{k} \chi_{J_{k}}(s)
$$

$\chi_{J_{k}}$ being the indicator function of the interval $J_{k}=\left[a_{k}, b_{k}\right] \leqq(-\infty, t]$. Then by definition (cf. Doob [5, p. $427(2.1)])^{5}$

$$
g=\sum_{k=1}^{n} c_{k}\left(\xi_{b_{k}}-\xi_{a_{k}}\right) \text {. }
$$

From (6.3) it is clear that $g \in \mathscr{N}_{t}$. Next suppose $c \in L_{2}(-\infty, t]$, and $c=\lim _{n \rightarrow \infty} c^{(n)}$, where $c^{(n)}$ is a step-function. Then by definition

${ }^{5} \mathrm{We}$ note that from $6.4(\mathrm{c})$ it follows that $\xi_{t-}=\xi_{t}=\xi_{t+},-\infty<t<\infty$. 


$$
g=\lim _{n \rightarrow \infty} \int_{-\infty}^{t} c^{(n)}(s) d \xi_{s} \in \mathscr{N}_{t},
$$

since $\mathscr{N}_{t}$ is closed. Thus $\mathscr{N}_{t}^{(\xi)} \subseteq \mathscr{N}_{t}$.

We may sum up the main results established so far as follows:

6.7 Theorem. (Wold Decomposition I) Let $\left(f_{t},-\infty<t<\infty\right)$ be a weakly stationary, mean-continuous, non-deterministic S.P. with shift group $\left(U_{t},-\infty<t<\infty\right)$. Let $h_{0}^{\prime}$ be the $0 t h$ normalized innovation of the associated discrete process, and let

$$
h_{t}=U_{t}\left(h_{0}^{\prime}\right), \quad \xi_{t}=h_{t}-h_{0}+\int_{0}^{t} h_{s} d s, \quad-\infty<t<\infty .
$$

Then (a) $\mathscr{M}_{t}=\mathscr{N}_{t}+\mathscr{M}_{-\infty}, \mathscr{N}_{t} \perp \mathscr{M}_{-\infty},-\infty<t<\infty$, where $\mathscr{N}_{t}=$ $\subseteq\left(h_{s}\right)_{s \leqq t}$ is the past and present of $h_{t}$;

(b) the $\xi_{t}$-process has stationary, orthogonal increments such that $\left|\xi_{t}-\xi_{s}\right|^{2}=|t-s| ;$ moreover, $\mathscr{N}_{t}=\mathfrak{S}\left(\xi_{\sigma}-\xi_{\tau}\right)_{\sigma, \tau \leqq t}$, i.e. $\mathscr{N}_{i}$ is the set of all stochastic integrals $\int_{-\infty}^{t} c(s) d \xi_{s}$ with $c \in L_{2}(-\infty, t]$.

6.8 Uniquenses Theorem. Let $\left(\eta_{t},-\infty<t<\infty\right)$ be any process with the following properties:

(i) it has orthogonal increments such that

$$
\left|\eta_{b}-\eta_{a}\right|^{2}=|b-a|, \quad-\infty<a, b<\infty \text {, and } \eta_{0}=0
$$

(ii) $U_{t}\left(\eta_{b}-\eta_{a}\right)=\eta_{b+t}-\eta_{a+t},-\infty<a, b, t<\infty$

(iii) $S\left(\eta_{\sigma}-\eta_{\tau}\right)_{\sigma, \tau \leqq 0}=\mathscr{M}_{-\infty}^{\perp} \cap \mathscr{M}_{0}$.

Then $\eta_{t}=e^{i \alpha} \xi_{t}$, where $\xi_{t}$ is as in 6.7 , and $\alpha$ is some real number.

Proof. Our proof of this result is essentially that given by Hanner [9, p. 175-176]. Since our treatments and notations differ, we may indicate the main steps. We first show that

$$
\mathfrak{S}\left(\eta_{\sigma}-\eta_{\tau}\right)_{\sigma, \tau \leqq b}=\mathscr{N}_{b}, \quad \mathfrak{S}\left(\eta_{\sigma}-\eta_{\tau}\right)_{a \leqq \sigma, \tau \leqq b}=\mathscr{N}_{a}^{\frac{1}{a}} \cap \mathscr{N}_{b},
$$

where $\mathscr{N}_{b}$ is an in 6.7(a). It follows from 6.7(b) that $\xi_{b}-\xi_{a}=\int_{a}^{b} f_{a, b}(s) d \eta_{s}$. By piecing together the functions $f_{n, n+1},-\infty<n<\infty$, we can define a function $f$ on $(-\infty, \infty)$ such that

$$
\xi_{b}-\xi_{a}=\int_{a}^{b} f(s) d \eta_{s}, \quad a<b .
$$

Using the fact that $\xi_{b}-\xi_{a}=U_{h}\left(\xi_{b-h}-\xi_{a-h}\right)$, we can show that $f$ is essentially constant-valued on $(-\infty, \infty)$. From this the desired result is immediate. 
An immediate corollary of Theorem 6.7 is the cannonical decomposition of the vector $f_{t}$ itself:

6.9 Corollary. (Wold Decomposition II) With the hypothesis of Theorem 6.7 we have

(a) $f_{t}=u_{t}+v_{t}, \quad u_{t}=\left(f_{t} \mid \mathscr{N}_{t}\right), \quad v_{t}=\left(f_{t} \mid \mathscr{M}_{-\infty}\right)$;

(b) the $u_{t}$-process in (a) is a one-sided moving average, i.e.

$$
u_{t}=\int_{0}^{\infty} c(s) d_{s} \xi_{t-s}, \quad-\infty<t<\infty, \text { where } c \in L_{2}[0, \infty) .
$$

and $\mathfrak{S}\left(u_{s}\right)_{s \leqq t}=\mathscr{N}_{t},-\infty<t<\infty$;

(c) the $v_{t}$-process is deterministic, and $\mathcal{S}\left(v_{s}\right)_{s \leqq t}=\mathscr{M}_{-\infty}$, for $-\infty<t<\infty$.

7. Purely non-deterministic stochastic processes. We call a weakly stationary S.P. purely non-deterministic, if and only if $\mathscr{C}_{-\infty}=\{0\}$. For completeness we state here the anologue of a theorem given by Kolmogorov for discrete parameter processes:

7.1 THEOREM. For any weakly stationary, mean-continuous stochastic process $\left(f_{t},-\infty<t<\infty\right)$ the following conditions are equivalent:

(i) $\left(f_{t},-\infty<t<\infty\right)$ is purely non-deterministic;

(ii) $\left(f_{t},-\infty<t<\infty\right)$ is a one-sided moving average:

$$
f_{t}=\int_{0}^{\infty} c(s) d_{s} \xi_{t-s}, \quad c \in L_{2}[0, \infty],
$$

$\left(\xi_{s},-\infty<s<\infty\right)$ being a process with stationary and orthogonal increments such that $\left|\xi_{b}-\xi_{a}\right|^{2}=|b-a|$;

(iii) $\lim _{t \rightarrow \infty}\left(f_{0} \mid \mathscr{M}_{-t}\right)=0$.

Proof. The proof runs parallel to that in the discrete case and is omitted.

It follows from Corollary 6.9 and Theorem 7.1 that every weakly stationary, mean-continuous, non-deterministic S.P. $\left(f_{t},-\infty<t<\infty\right)$ can be decomposed in the form $f_{t}=u_{t}+v_{t}$, where the $u_{t}$-process is purely non-deterministic, the $v_{t}$-process is deterministic, and all three processes have the same shift group $\left(U_{t},-\infty<t<\infty\right)$. We shall refer to the $u_{t}$-and $v_{t}$-processes as the purely non-deterministic part and the deterministic part of the $f_{t}$-process. With an obvious notation, we have

$$
\begin{aligned}
& \mathscr{H}_{t}=\mathscr{M}_{t}^{(u)}+\mathscr{M}_{t}^{(v)}, \quad \mathscr{M}_{\infty}^{(u)} \perp \mathscr{M}_{\infty}^{(v)} \\
& \mathscr{M}_{t}^{(u)}=\mathscr{N}_{t}, \quad \mathscr{M}_{t}^{(v)}=\mathscr{M}_{-\infty} .
\end{aligned}
$$

Now let $\left(u_{n}^{\prime}\right)_{-\infty}^{\infty},\left(v_{n}^{\prime}\right)_{-\infty}^{\infty}$ be the purely non-deterministic and determin- 
istic parts of the discrete process $\left(f_{n}^{\prime}\right)_{-\infty}^{\infty}$ associated with $\left(f_{t},-\infty<t<\infty\right)$. Then by 6.9(a), 4.4(c), and (5.8)

$$
v_{0}=\left(f_{0} \mid \mathscr{C}_{-\infty}\right)=\left(f_{0}^{\prime} \mid \mathscr{M}_{-\infty}^{\prime}\right)=v_{0}^{\prime},
$$

and therefore

$$
u_{0}=f_{0}-v_{0}=f_{0}^{\prime}-v_{0}^{\prime}=u_{0}^{\prime} .
$$

Moreover, the shift operator $V$ of the $u_{n}^{\prime}$, $v_{n}^{\prime}$-processes is the Cayley transform of $H$, where $i H$ is the infinitesimal generator of the shift group $\left(U_{t},-\infty<t<\infty\right)$ of the $u_{t^{-}}, v_{t}$-processes. We can thus assert the following:

7.2 COROLlaRY. If $\left(f_{n}^{\prime}\right)^{\infty}$ is the discrete process associated with the weakly stationary, mean-continuous, non-deterministic S.P. $\left(f_{t}\right.$, $-\infty<t<\infty)$, then the purely non-deterministic and deterministic parts of $\left(f_{n}^{\prime}\right)_{-\infty}^{\infty}$ are the discrete processes associated with the deterministic and purely non-deterministic parts of $\left(f_{t},-\infty<t<\infty\right)$.

\section{REFERENCES}

1. K. Balagangadharan, The prediction theory of stationary random distributions, Memoirs of the College of Sci., University of Kyoto, series A, 33 (1960), 243-256.

2. A. Beurling, On two problems concerning linear transformations in Hilbert space, Acta Mathematica, 81 (1949), 239-255.

3. J. L. B. Cooper, One-parameter semi-groups of isometric operators in Hilbert space, Ann. Math., 48 (1947), 827-842.

4. H. Cramer, On the linear prediction problem for certain stochastic processes, Ark. for Mat., 4 (1959), 45-54.

4'. - On some classes of non-stationary stochastic processes, Proc. Fourth Berkeley Symposium of Mathematical Statistics and Probability, Berkeley (1960), 57-78.

$4^{\prime \prime}$. — On the structure of purely non-deterministic stochastic processes, Ark. for Mat., 4 (1961), 249-266.

5. J. L. Doob, Stochastic Processes, New York, 1953.

6. N. Dunford and J. Schwarz, Linear Operators, Part I, New York, 1958.

7. I. M. Gelfand, Stationary random processes (Russian), C. R. (Doklady), Acad. Sci. U.R.S.S., 100 (1955), 853-856, 208 (1961), 102-112.

8. P. Halmos, Shifts of Hilbert spaces, Crelle's Journal, 208 (1961), 102-112.

9. O. Hanner, Deterministic and non-deterministic stationary random processes, Ark. for Mat., 1 (1950), 161-177.

10. H. Helson and D. Lowdenslager, Prediction theory and Fourier series in several variables, Acta Mathematica, 99 (1958), 165-202.

11. E. Hille and R. S. Phillips, Functional Analysis and Semigroups, Providence, 1957.

12. K. Ito, Stationary random distributions, Memoirs of the College of Sci., Univ. of Kyoto, Series A, 28 (1953), 209-223.

13. K. Karhunen, Über lineare Methoden in der Warhrscheinlickeitsrechnung, Am. Acad. Sci. Fennicae, A, I, 37 (1947).

14. P. Lax, Translation invariant subspaces, Acta Mathematica, 101 (1959), 163-178.

15. P. Masani, Shift invariant spaces and prediction theory, Acta Mathematica, 107 (1962), 275-290. 
16. - On isometric flows on Hilbert space. Bull. Amer. Math. Soc., 68 (1962), 624-632.

17. F. Riesz and B. Sz.-Nagy, Functional Analysis, Translated from 2nd French Ed., New York, 1959.

18. G. Sansone, Orthogonal Polynomials, New York, 1959.

19. M. H. Stone, Linear Transformations in Hilbert Space, New York, 1932.

20. N. Wiener and A. Wintner, Random time, Nature, 181 (1958), 561-562.

INDIANA UNIVERSITY 


\section{PACIFIC JOURNAL OF MATHEMATICS}

\section{EDITORS}

\author{
Ralph S. Phillips \\ Stanford University \\ Stanford, California \\ M. G. Arsove \\ University of Washington \\ Seattle 5 , Washington
}

\author{
A. L. Whiteman \\ University of Southern California \\ Los Angeles 7, California \\ Lowell J. Paige \\ University of California \\ Los Angeles 24, California
}
E. F. BECKENBACH
D. DERRY
ASSOCIATE EDITORS
T. M. CHERRY
M. OHTSUKA
H. L. ROYDEN
E. G. STRAUS
E. SPANIER
F. WOLF

\section{SUPPORTING INSTITUTIONS}

\author{
UNIVERSITY OF BRITISH COLUMBIA \\ CALIFORNIA INSTITUTE OF TECHNOLOGY \\ UNIVERSITY OF CALIFORNIA \\ MONTANA STATE UNIVERSITY \\ UNIVERSITY OF NEVADA \\ NEW MEXICO STATE UNIVERSITY \\ OREGON STATE UNIVERSITY \\ UNIVERSITY OF OREGON \\ OSAKA UNIVERSITY \\ UNIVERSITY OF SOUTHERN CALIFORNIA
}

\author{
STANFORD UNIVERSITY \\ UNIVERSITY OF TOKYO \\ UNIVERSITY OF UTAH \\ WASHINGTON STATE UNIVERSITY \\ UNIVERSITY OF WASHINGTON \\ AMERICAN MATHEMATICAL SOCIETY \\ CALIFORNIA RESEARCH CORPORATION \\ SPACE TECHNOLOGY LABORATORIES \\ NAVAL ORDNANCE TEST STATION
}

Mathematical papers intended for publication in the Pacific Journal of Mathematıcs should be typewritten (double spaced), and the author should keep a complete copy. Manuscripts may be sent to any one of the four editors. All other communications to the editors should be addressed to the managing editor, L. J. Paige at the University of California, Los Angeles 24, California.

50 reprints per author of each article are furnished free of charge; additional copies may be obtained at cost in multiples of 50 .

The Pacific Journal of Mathematics is published quarterly, in March, June, September, and December. Effective with Volume 13 the price per volume (4 numbers) is $\$ 18.00$; single issues, $\$ 5.00$. Special price for current issues to individual faculty members of supporting institutions and to individual members of the American Mathematical Society: $\$ 8.00$ per volume; single issues $\$ 2.50$. Back numbers are available.

Subscriptions, orders for back numbers, and changes of address should be sent to Pacific Journal of Mathematics, 103 Highland Boulevard, Berkeley 8, California.

Printed at Kokusai Bunken Insatsusha (International Academic Printing Co., Ltd.), No. 6, 2-chome, Fujimi-cho, Chiyoda-ku, Tokyo, Japan.

PUBLISHED BY PACIFIC JOURNAL OF MATHEMATICS, A NON-PROFIT CORPORATION

The Supporting Institutions listed above contribute to the cost of publication of this Journal, but they are not owners or publishers and have no responsibility for its content or policies. 


\section{Pacific Journal of Mathematics}

\section{Vol. 12 , No. 4 \\ April, 1962}

Tsuyoshi Andô, On fundamental properties of a Banach space with a cone ..... . 1163

Sterling K. Berberian, A note on hyponormal operators ................ 1171

Errett Albert Bishop, Analytic functions with values in a Frechet space . . . . . . . 1177

(Sherman) Elwood Bohn, Equicontinuity of solutions of a quasi-linear equation ............................................ 1193

Andrew Michael Bruckner and E. Ostrow, Some function classes related to the class of convex functions . . . . . . . . . . . . . . . . . . . . . . . . 1203

J. H. Curtiss, Limits and bounds for divided differences on a Jordan curve in the complex domain . . ................................. 1217

P. H. Doyle, III and John Gilbert Hocking, Dimensional invertibility . . . . . . . . 1235

David G. Feingold and Richard Steven Varga, Block diagonally dominant matrices and generalizations of the Gerschgorin circle theorem ................. 1241

Leonard Dubois Fountain and Lloyd Kenneth Jackson, A generalized solution of the boundary value problem for $y^{\prime \prime}=f\left(x, y, y^{\prime}\right) \ldots \ldots \ldots \ldots \ldots \ldots \ldots \ldots \ldots$

Robert William Gilmer, Jr., Rings in which semi-primary ideals are primary. . . . . 1273

Ruth Goodman, K-polar polynomials .......................... 1277

Israel Halperin and Maria Wonenburger, On the additivity of lattice completeness ........................................... 1289

Robert Winship Heath, Arc-wise connectedness in semi-metric spaces . . . . . . . . 1301

Isidore Heller and Alan Jerome Hoffman, On unimodular matrices . . . . . . . . . . . 1321

Robert G. Heyneman, Duality in general ergodic theory . . . . . . . . . . . . . . . 1329

Charles Ray Hobby, Abelian subgroups of p-groups . . . . . . . . . . . . . . . 1343

Kenneth Myron Hoffman and Hugo Rossi, The minimum boundary for an analytic

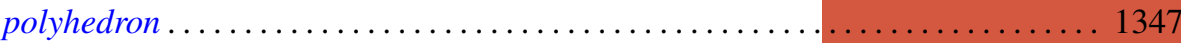

Adam Koranyi, The Bergman kernel function for tubes over convex cones ........ 1355

Pesi Rustom Masani and Jack Max Robertson, The time-domain analysis of a continuous parameter weakly stationary stochastic proces.

William Schumacher Massey, Non-existence of almost-complex structures on

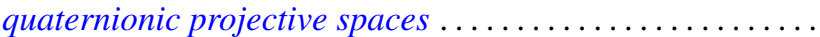

Deane Montgomery and Chung-Tao Yang, A theorem on the action of $\mathrm{SO}(3) \ldots . .1385$

Ronald John Nunke, A note on Abelian group extensions . . . . . . . . . . . . . 1401

Carl Mark Pearcy, A complete set of unitary invariants for operators generating finite $W^{*}$-algebras of type $I$

Edward C. Posner, Integral closure of rings of solutions of linear differential equations.

Duane Sather, Asymptotics. III. Stationary phase for two parameters with an application to Bessel functions.

J. Śladkowska, Bounds of analytic functions of two complex variables in domains

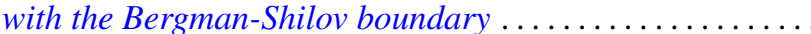

Joseph Gail Stampfli, Hyponormal operators .

George Gustave Weill, Some extremal properties of linear combinations of kernels

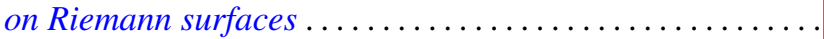

